\title{
The connection between prestellar cores and filaments in the Aquila molecular cloud complex
}

\author{
Vera Könyves $^{1}$ and Philippe André ${ }^{1}$ \\ ${ }^{1}$ Laboratoire AIM, CEA/DSM-CNRS-Université Paris Diderot, IRFU/Service \\ d'Astrophysique, CEA Saclay, 91191 Gif-sur-Yvette, France \\ emails: vera.konyves@cea.fr, philippe.andre@cea.fr
}

\begin{abstract}
One of the main scientific goals of the Herschel Gould Belt survey is to elucidate the physical mechanisms responsible for the formation and evolution of prestellar cores in molecular clouds. In the $\sim 11 \mathrm{deg}^{2}$ field of Aquila imaged with Herschel/PACS-SPIRE at 70-500 $\mu \mathrm{m}$, we have identified a complete sample of 651 starless cores, 446 of them are gravitationally-bound candidate prestellar cores. Our Herschel observations also provide an unprecedented census of filaments in the Aquila cloud and suggest an intimate connection between these filaments and the formation process of prestellar cores. Indeed, a strong correlation is found between their spatial distributions. These Herschel findings support a filamentary paradigm for the early stages of star formation, where the cores result from the gravitational fragmentation of the densest filaments.
\end{abstract}

Keywords. stars: formation, ISM: clouds, ISM: structure, ISM: individual objects (Aquila Rift complex), submillimeter

The Herschel Gould Belt survey (André et al.2010) observations in the Aquila cloud ( $d \sim$ $260 \mathrm{pc}$ ) have provided an essentially complete census of prestellar cores and dense filaments†. The cores have been identified using getsources (Men'shchikov et al.2012), and the filaments have been traced and compared by several methods (see Könyves et al.2010, 2015).

Fig. 1 shows the filamentary structure of the Aquila cloud, traced by the curvelet transform component (Starck et al.2003) of the column density map. Given the typical filament width of $\sim 0.1 \mathrm{pc}$ (e.g., Arzoumanian et al.2011), this curvelet map provides information on the mass per unit length along the filaments (André et al.2010). The white areas highlight regions where this stability parameter exceeds half the critical line mass $M_{\text {line,crit }}=2 c_{\mathrm{s}}^{2} / G$ (Inutsuka \& Miyama 1997) and the filaments are thus likely gravitationally supercritical (see Könyves et al.2015).

A high fraction $(\sim 90 \%)$ of our prestellar cores are above a background column density corresponding to $A_{\mathrm{V}} \sim 7$, while a similar high portion $(\sim 75 \%)$ of these cores lie within the densest filaments with supercritical mass per unit length. As seen in Fig. 1 a strong correlation is found between the spatial distribution of prestellar cores and the densest filaments above $A_{\mathrm{V}}^{b g} \sim 7$, or above an approximately corresponding line mass of $\sim 16 M_{\odot} / \mathrm{pc}$ along the filaments (André et al.2014, Könyves et al.2015).

Comparing the statistics of cores and filaments with the number of young stellar objects found by Spitzer in the same complex (Dunham et al.2013, Allen et al., in prep.), we also infer a typical timescale of $\sim 1 \mathrm{Myr}$ for the formation and evolution of both prestellar cores and filaments.

In summary, our Herschel results support a scenario in which the formation of solar-type stars occurs in two main steps (André et al.2014): 1) the dissipation of large-scale magnetohydrodynamic waves generates quasi-universal filaments in the cold ISM, 2) the densest filaments fragment into prestellar cores by gravitational instability.

\section{References}

André, P., Men'shchikov, A., Bontemps, S., et al. 2010, A\&A, 518, L102+

$\dagger$ Maps and catalogs derived from our Herschel data can be found at the Herschel Gould Belt Survey Archive, http://gouldbelt-herschel.cea.fr/archives. 


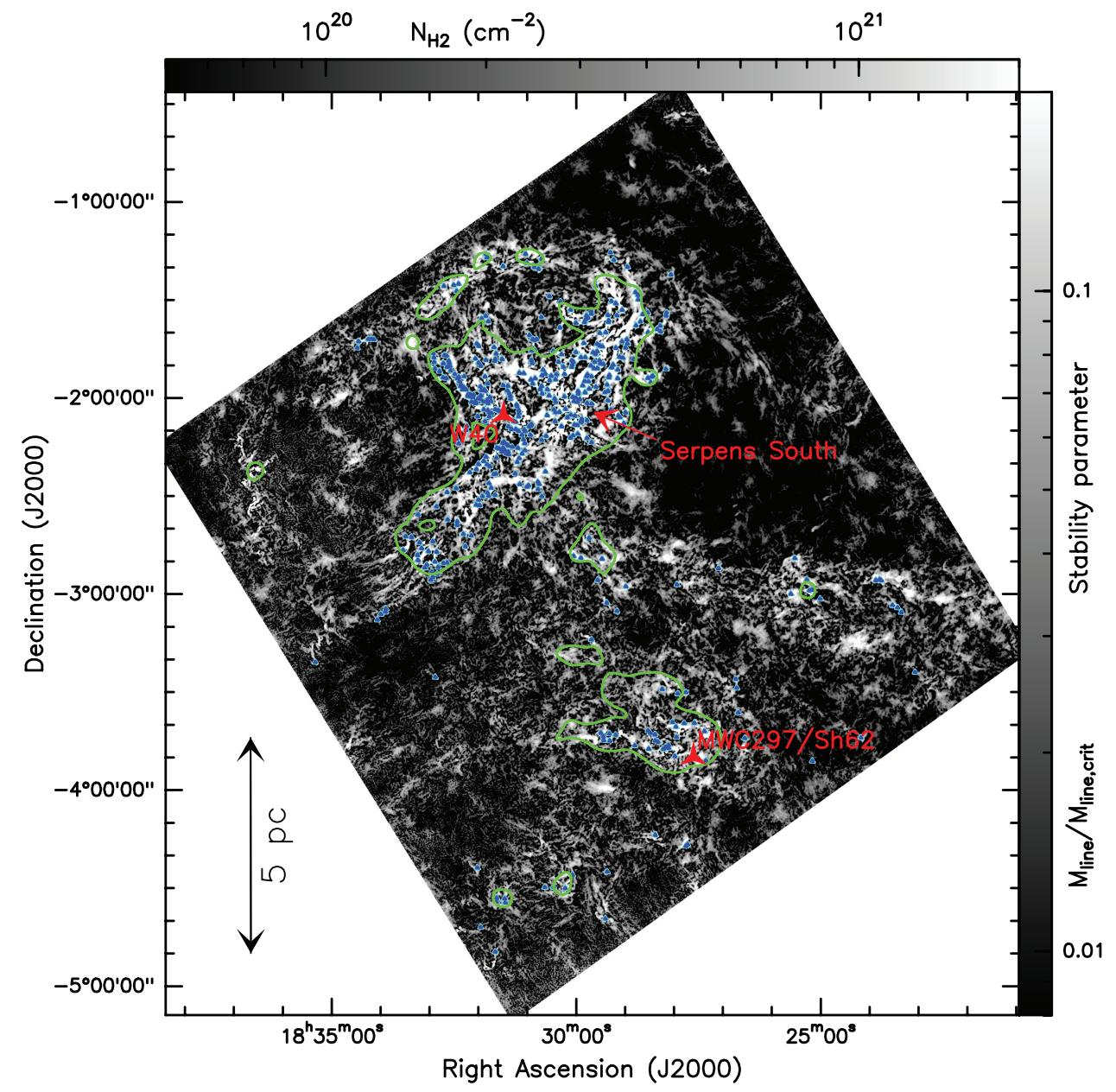

Figure 1. Spatial distribution of the prestellar cores (blue triangles) and the network of filaments in the Aquila cloud as traced by the curvelet component of the Herschel column density map. The likely gravitationally unstable filaments are highlighted in white, and the green contours correspond to $A_{\mathrm{V}} \sim 7$ (Könyves et al.2015).

André, P., Di Francesco, J., Ward-Thompson, D., et al. 2014, in PPVI, ed. H. Beuther et al., 27 Arzoumanian, D., André, P., Didelon, P., et al. 2011, A\&A, 529, L6

Dunham, M. M., Arce, H. G., Allen, L. E., et al. 2013, AJ, 145, 94

Inutsuka, S.-I. \& Miyama, S. M. 1997, ApJ, 480, 681

Könyves, V., André, P., Men'shchikov, A., et al. 2010, A\&\&A, 518, L106+

Könyves, V., André, P., Men'shchikov, A., et al. 2015, A\&SA, in press, arXiv:1507.05926

Men'shchikov, A., André, P., Didelon, P., et al. 2012, A\&GA, 542, A81

Starck, J. L., Donoho, D. L., \& Candès, E. J. 2003, A\&A, 398, 785 